\title{
L'apprentissage et le développement des compétences scripturales méritent une réflexion
}

Ismail Bou-serdane

Université de Sherbrooke, Québec, Canada

Faculté d'éducation

Département de pédagogie

\begin{abstract}
Résumé
Plusieurs élèves et étudiants au Maroc du niveau primaire, secondaire, collégial et universitaire présentent des difficultés en écriture, en langue arabe et en langues étrangères. Nous savons d'ailleurs qu'écrire est l'un des objets les plus complexes de l'apprentissage (Barratte, 2007). Cette complexité confère à l'enseignant de langue une responsabilité majeure. Il ne s'agit plus d'enseigner les aspects linguistiques de la langue, mais aussi les processus cognitif, métacognitif et affectif mis en œuvre pour produire le discours. Actuellement, la recherche démontre que les pratiques enseignantes ont une incidence favorable sur le succès des élèves dans leur apprentissage de la lecture et de l'écriture. Dès lors, changer sa manière de voir l'enseignementapprentissage d'écrire au Maroc est devenu primordiale.
\end{abstract}

Mots clés: difficultés d'écriture - apprentissage de l'écriture - pratiques enseignantes compétences scripturales - théorie de l'apprentissage socioconstructiviste - intervention.

\section{Learning and development scriptural skills deserve a reflection}

\begin{abstract}
Several pupils and students in Morocco of the primary, secondary, collective and university level present difficulties in writing, in Arabic language and in foreign languages. We know moreover that to write is one of the most complex objects of the apprenticeship (Barratte, on 2007). This complexity confers to the teacher of language a major responsibility. It is not any more a question of teaching the linguistic aspects of the language, but also the processes cognitive, metacognitive and emotional implemented to produce the speech. At present, the research demonstrates that the teaching practices have a favorable incidence on the success of the pupils in their apprenticeship of the writing. From then on, changing his view teaching and apprenticeship of writing in Morocco has become paramount
\end{abstract}

Keywords: Difficulties of writing - writing apprenticeship - teaching practices - scriptural skills theory of the apprenticeship socioconstructiviste - intervention.

\section{Introduction}

Depuis plusieurs années, l'apprentissage de la langue écrite occupe une place majeure dans les réformes éducatives au Maroc. Cette importance donnée à l'écriture vient du fait que celle-ci est 
devenue une pratique fondamentale, non seulement dans la vie privée, mais aussi dans la vie sociale et professionnelle. Toutefois, dans les rapports nationaux (Commission spéciale éducation-formation, 1999 ; association marocain des enseignants de français, 2010) concernant les compétences scripturales, les élèves et les étudiants éprouvent des difficultés majeures à produire des textes cohérents et sans trop d'erreurs. Pourtant, écrire est l'un des objets les plus complexes de l'apprentissage (Barratte, 2007). Cette complexité confère à l'enseignant de langue une responsabilité majeure. En effet, actuellement, l'apprentissage de l'écriture et sa maîtrise ne reposent plus seulement sur l'aspect linguistique de la langue (orthographe, syntaxe, lexique), mais ils s'appuient aussi sur l'apprentissage de la langue en tant qu'organisation discursive décontextualisée qui, malgré la linéarité figurale qu'elle suppose, implique une hiérarchisation, des explications, un raisonnement causal, une logique particulière. Un texte en effet doit progresser régulièrement, présenter une grande cohérence et rendre compte le plus explicitement possible des intentions illocutoires de son auteur (Olson, 1998).

Changer sa manière de voir l'apprentissage de l'écriture est donc devenu primordial. C'est sur toutes les dimensions de l'écriture qu'il importe d'agir. L'objectif de cet article est de mettre en lumière l'apprentissage et le processus de développement des compétences scripturales. Elle vise également à proposer une démarche ${ }^{1}$ pour envisager autrement l'enseignement-apprentissage de l'écriture à travers une modélisation socioconstructiviste de l'ordre du scriptural. Cette démarche tiendra compte des récentes recherches en didactique des langues. Pour ce faire, dans un premier temps, nous présentons dans la problématique de cet article la situation actuelle de l'enseignement-apprentissage de l'écriture au Maroc, en nous basant sur des recherches et des rapports nationaux. De cette situation découle le problème de recherche. Nous exposons ensuite, en recension des écrits, les résultats des recherches sur les incidences des pratiques enseignantes sur le processus d'apprentissage de l'écriture auprès d'élèves. Pour envisager une démarche d'enseignement pouvant favoriser l'apprentissage de l'écriture, nous spécifions, dans un deuxième temps, les particularités de l'écriture, et ce qui la distingue de l'oral. Cela nous permettra de mieux comprendre ce qu'est l'écriture, les apprentissages qu'elle implique, les processus mis en œuvre par le scripteur (élève) dans une situation d'écriture et les compétences qui y sont associées.

\section{Problématique}

Au Maroc, depuis plusieurs années, la qualité de la langue écrite en arabe ou en langues étrangères des élèves et étudiants des niveaux primaire, secondaire et universitaire suscite plusieurs débats. Enseignants, parents, inspecteurs, responsables de l'éducation, journalistes et politiciens, tous dénoncent la qualité de la langue écrite des élèves et des étudiants. Dans cette perspective, Kesbi (2011) déclare que les textes rédigés en arabe classique constituent un handicap de taille pour les élèves. D'autres recherches révèlent que les élèves et les étudiants ne réussissent pas encore à écrire des textes cohérents et sans trop d'erreurs en langues étrangère ${ }^{2}$,

\footnotetext{
${ }^{1}$ Le terme «démarche d'enseignement-apprentissage» est retenu dans cet article pour désigner le cheminement structuré de l'enseignant (son itinéraire, la succession des actions) pour permettre à l'élève de s'engager dans un processus d'apprentissage afin d'établir un rapport au savoir approprié et de poursuivre ainsi l'atteinte des objectifs.

${ }^{2}$ L'expression langue étrangère réfère au statut de la langue française, anglaise et espagnole au Maroc
} 
même après maintes années de scolarisation. Arraichi (2002), dans une recherche sur les productions écrites en français menée auprès d'élèves du baccalauréat scientifique dans le monde rural en 1998, souligne que ces élèves éprouvent de grandes difficultés au niveau de la maîtrise de la langue, de la compétence scripturale ${ }^{3}$, de la structuration du texte et de l'autonomie ${ }^{4}$ des idées et du style. Dans le même ordre d'idées, l'association marocaine des enseignants du français (2010) affirme que la production écrite pour les élèves constitue un moment difficile. Les élèves n'éprouvent aucun plaisir à écrire ni à apprendre à écrire. Toujours selon cette association (Ibid.), ces difficultés éprouvées par les élèves, ainsi que le retard scolaire qu'ils accusent à l'arrivée au secondaire, posent des questions quant à leur réussite et leur persévérance.

Sur un autre plan, les contenus enseignés et les pratiques enseignantes mises en place en classe sont pointés du doigt pour tenter d'expliquer les piètres performances scripturales des élèves. La charte nationale de l'éducation et de la formation de la commission spéciale éducation-formation (1999), le rapport du conseil supérieur de l'enseignement (2008) et le programme d'urgence du ministère de l'Éducation nationale (2008) soulignent que les enseignants utilisent toujours un mode d'intervention en classe basé sur des activités répétitives, traditionnelles et décontextualisées. Selon Reuter (1996), dans ce genre de pratiques enseignantes, l'enseignementapprentissage de l'écriture se présente comme une synthèse des autres enseignements disciplinaires, notamment l'enseignement de l'orthographe, de la syntaxe, de la conjugaison et du vocabulaire. Dans ce contexte, c'est aux élèves d'apprendre, par eux-mêmes, et d'intégrer ces enseignements. L'enseignement-apprentissage de l'écriture ne peut donc se réduire à la seule maîtrise de codes linguistiques et sémantiques. Ce genre de démarches à manifester ses limites et fait ressentir la nécessite de transformations importantes (Reuter, Ibid.)

Depuis quelques années, plusieurs recherches ont démontré le besoin de changer les pratiques enseignantes pour aider les élèves dans leur apprentissage de l'écriture. Des recherches relatives à l'observation de pratiques enseignantes (Prenoveau, 2007; Strickland, Ganske et Monroe, 2009) décrivent la complexité du rapport qu'entretient l'enfant avec l'écrit tout au long du primaire. Ces recherches affirment que des pratiques pédagogiques efficaces pourraient faciliter le développement des compétences à lire et à écrire chez les élèves. D'autres recherches (Adad et Richard-Principalli, 2007; Giasson, 2011) soulignent que les pratiques gagnantes 5 en enseignement reposent principalement sur les compétences des enseignantes. Reuter $(2000,2011)$ et Routman (2009) mentionnent également qu'il existe un lien entre les performances des élèves en écriture et en lecture et des pratiques enseignantes gagnantes. Ils considèrent que ces pratiques jouent un rôle important et peuvent aider les élèves, même ceux en difficulté, à vivre un apprentissage réussi de la lecture et de l'écriture. L'école marocaine est ainsi appelée à adopter une nouvelle vision pédagogicodidactique ${ }^{6}$ quant à l'enseignement-apprentissage afin d'améliorer les pratiques enseignantes au regard de l'apprentissage de l'écriture.

\footnotetext{
${ }^{3}$ La notion de compétence scripturale n'est pas définie par l'auteur.

${ }^{4}$ La notion d'autonomie n'est pas définie par l'auteur.

${ }^{5}$ Selon le mouvement québécois de la qualité, la pratique gagnante est une technique ou une méthode non encore normalisée, mais appliquée avec succès par une ou plusieurs organisations. Elle se caractérise par son aspect innovateur et par les résultats positifs qu'elle produit et qui ont un impact mesurable sur la performance de l'organisation.

${ }^{6}$ Le terme pédagogicodidactique renvoie à «la fonction didactique de structuration et de gestion des contenus» et à la «fonction pédagogique d'aide à la construction du savoir par la relation fonctionnelle et l'organisation des apprentissages» (Altet, 1997, p. 11-12).
} 



\section{Entre l'écriture et l'oral}

De prime abord, il convient de ne pas prendre le mot «écrire » au sens de «copier» ou de « tracer des signes graphiques ». En ce qui nous concerne, dans cet article, l'écriture est vue dans au sens d'une "activité d'énonciation». Écrire ou rédiger est donc un acte langagier de communication, un acte créatif, qui permet de verbaliser sa pensée sous une forme tangible et durable (Lentin, 1998). De ce fait, l'entrée dans l'écrit ${ }^{7}$ implique une rupture avec l'oralité (toujours inscrite dans l'interaction), Nous optons donc dans ce texte, pour la définition de Reuter (1996) selon laquelle:

L'écriture est une pratique sociale, historiquement construite, impliquant la mise en œuvre généralement conflictuelle de savoirs, de représentations, de valeurs, d'investissements et d'opérations, par laquelle un ou plusieurs sujets visent à (re)produire du sens, linguistiquement structuré, à l'aide d'un outil, sur un support conservant durablement ou provisoirement de l'écrit, dans un espace socio-institutionnel donné (p. 58).

Selon cette définition, l'écriture signifie plus que l'appropriation d'un code linguistique. Il s'agit d'entrer dans «l'ordre scriptural » (Dabène, 1987). Par ce terme, Dabène (Ibid.) fait référence à la capacité de produire du sens par l'écrit, sans qu'il y ait transit par l'oral, par l'appropriation d'outils sémiotiques et de matériel écrit. C'est également le développement et le contrôle de son activité langagière «en prenant son texte comme un objet qu'on crée, analyse, commente, structure, manipule et transforme » (Chartrand, 2000a, p. 26).

L'oral et l'écrit se distinguent donc d'abord selon leurs conditions d'utilisation. L'oral est associé à ce qui est immédiat et en situation, c'est-à-dire que la présence de l'interlocuteur est médiate dans l'espace et dans le temps. Cela a pour impact qu'il n'y a pas de délai entre la production du message et sa réception, sans compter les éléments du paralangage nécessaires à la compréhension du message (geste, mimique par exemple), ajoutés pour favoriser l'économie de moyens linguistiques. L'écrit, quant à lui, est différé dans le temps et hors situation. Puisque le locuteur est absent et qu'il existe un délai entre la production et la réception du message, le cadre spatiotemporel doit être précisé. En effet, le scripteur ne profite pas des réactions immédiates de l'interlocuteur. Les conditions de production constituent une autre distinction entre l'oral et l'écrit. À l'oral, le message est construit et émis devant l'interlocuteur, ce qui permet de modifier, de restructurer et de corriger le message au besoin, mais sans revenir en arrière, c'est-à-dire au début de la production du message. Toutefois, l'écrit se caractérise par la régression, c'est-à-dire qu'il permet au scripteur de réfléchir et de construire son message, tout en ayant la possibilité de revenir en arrière aussi souvent qu'il le désire. Cela donne à la communication écrite un caractère plus fini et plus continu que l'oral (Riegel, Pellat et Rioul, 1998). Ainsi, entrer dans le monde de l'écriture est bien différent, voire plus difficile, que dans le monde de l'oral. «L'entrée dans l'ordre scriptural est associée à une transformation du fonctionnement langagier naturel, c'est une révolution cognitive » (Chartrand, 2000a, p. 26).

\footnotetext{
${ }^{7}$ Dans cet article, le terme écriture est utilisé comme synonyme de l'écrit.
} 


\section{Processus et développement des compétences scripturales}

L'écriture figure parmi les objets d'apprentissage les plus complexes. Vygotsky (1985) a écrit, dans cette perspective, qu'en « ce qui concerne l'apprentissage de l'écriture, nous devons tenir un discours différent. Des recherches menées à ce propos et dont nous parlerons ailleurs ont démonté que ce processus ouvre une série de nouveaux cycles de développement d'une grande complexité » (p. 113-114). Le ministère de l'Éducation, de loisir et du sport du Québec (2008) rappelle que l'acte d'écrire est une tâche complexe :

c'est beaucoup plus qu'écrire un texte sans faute. Écrire constitue un acte complexe qui met en jeu à la fois des processus cognitifs et des représentations sociales. L'élève doit planifier sa démarche, mettre son texte en forme et le réviser. Il a donc beaucoup d'aspects à gérer en plus de la grammaire (p. 4).

On comprend donc que le processus mis en place par le scripteur en classe n'est pas un acte simple. Il suppose la mise en place d'une activité dans laquelle l'élève devra mettre à l'épreuve son organisation mentale. C'est cette activité mentale qui peut favoriser, selon Rey (2004), le développement de compétences de troisième degré ou des compétences complexes. Il s'agit là non seulement de choisir, mais aussi de combiner d'une façon cohérente les procédures de base dans des situations variées d'écriture. Pour cela, la didactique de l'écriture, telle qu'elle est pensée aujourd'hui, incite les enseignants à adopter des démarches d'enseignement-apprentissage travaillant les processus mis en ouvre dans l'acte scriptural, ainsi que les stratégies d'ordre cognitif et métacognitif. Ces stratégies permettent davantage au scripteur de tirer profit des multiples potentialités de l'écrit, de gérer les différentes situations d'écriture et de produire des textes appropriés, que de travailler l'aspect communicatif et normatif de la langue (Cansigno, Dezutter, Silva et Bleys, 2010). Bref, elles permettent le développement de compétences scripturales de troisième degré.

La compétence scripturale se définit comme étant «la capacité de produire du sens par l'écrit sans qu'il y ait transit par l'oral, par l'appropriation des outils sémiotiques et matériels de l'écrit; c'est développer le contrôle de son activité langagière en prenant son texte comme un objet qu'on crée, commente, structure, manipule et transforme » (Chartrand, 2000b, p. 26). D'après cette définition, il est possible de comprendre que tous les écrits ne s'inscrivent pas dans l'ordre scriptural, comme la retranscription des élèves des écrits de l'enseignant sur le tableau dans leur cahier ou le listage des écrits qui ne requièrent ni les connaissances ni les appropriations spécifiques à l'ordre scriptural. Néanmoins, l'écriture d'une planification ou la rédaction d'un message sont des types d'écrits qui engagent le sujet scripteur, car ils requièrent des connaissances non seulement linguistiques, mais aussi sémantiques, textuelles et syntaxiques. Elles nécessitent également des connaissances au regard du processus d'écriture, ainsi qu'une représentation assez juste de ses capacités.

La compétence scripturale est donc pluridimensionnelle. Elle implique non seulement des connaissances linguistiques (orthographe, syntaxe et lexique), mais aussi discursives (grammaire du texte), communicatives, cognitives et métacognitives (Cansigno et al., 2010). Ce sont ces connaissances en particulier qui permettent de développer une pensée critique lors de l'écriture sur un sujet donné. Travailler sur l'organisation des idées, sur la progression de l'information, sur 
la manière d'argumenter, c'est également réfléchir sur le contenu du texte, ce qui permet de construire des connaissances disciplinaires. La compétence scripturale tient compte également de deux autres particularités : celle de la variabilité ${ }^{8}$ et celle de l'extensivité ${ }^{9}$ selon l'appellation de Cansigno et al. (Ibid.). Cette compétence se développe lorsque des situations variées sont proposées aux élèves. Un élève ne peut manifester en classe les mêmes niveaux de maitrise dans différentes formes d'écrit associées à une situation. Il peut éprouver une certaine aisance en rédigeant une forme de texte et manifester des difficultés dans une autre. À chaque nouvelle situation (nouvelle forme de texte), il fera un nouvel apprentissage scriptural et développera alors une nouvelle compétence. Ainsi, cette compétence scripturale n'est pas figée dans le temps, mais elle se développe perpétuellement (Cansigno et al.,Ibid.). Sur ce point, lorsqu'on parle de la variabilité comme particularité de développement de compétences scripturales, on ne fait pas allusion aux variétés de textes qu'on présente aux élèves dans des activités d'enseignementapprentissage pour reconnaitre et nommer le type de texte. On ne fait allusion de même aux activités qui abrègent les types de texte en une catégorie d'unités linguistique. Selon Reuter (2000), cette manière de voir les choses «n'est cependant pas intéressante, car elle permet, au minimum, de distinguer des choix structurels possibles » (p. 120). Pour faire apprendre, selon le même auteur, à un élève à ne pas mettre les doigts dans les prises électriques, il est possible de recourir à plusieurs types de texte : 1) le texte injectif (sur le mode de l'interdiction); 2) le texte narratif (histoire d'un enfant qui, malgré l'interdiction de ses parents, a reçu une décharge après avoir mis ses doigts dans une prise électrique); 3 ) le texte explicatif (explication d'une prise et du courant électrique) et; 4) le texte argumentatif (aide à l'argumentation pour modifier l'intention de mettre son doigt dans la prise et exprimer ses opinions). Bref, il importe de contextualiser et de donner du sens aux apprentissages en classe.

En nous basant sur la description du processus et des compétences scripturales développées cidessus, nous pouvons dire que les pratiques enseignantes doivent prendre en considération ce qui est de l'ordre d'une compétence scripturale, plutôt que d'une pratique artificielle traduisant fidèlement la pensée des enseignants et tenant peu compte des écrits intermédiaires de leurs élèves (Blazer, 2007). Dès lors, la mise en place d'une démarche favorisant l'apprentissage de l'écriture selon un processus de développement des compétences est primordiale.

\section{Vers des pratiques enseignantes pouvant favoriser la réussite de l'apprentissage et le développement de compétences scripturales}

La recherche scientifique en didactique des langues, comme nous l'avons présenté dans la problématique, accorde actuellement une importance aux conceptions socioconstructivistes de l'apprentissage (Vygotsky, 1978). La pensée socioconstructiviste s'intéresse, comme Piaget (1969) le mentionne, au processus de construction du savoir. Toutefois, contrairement à ce dernier qui considère la pensée d'un point de vue individuel, les socioconstructivistes postulent que le développement cognitif se construit dans l'interaction sociale et au sein d'un contexte sociohistorique. Pour eux, la pensée n'est pas individuelle, mais socioculturelle. Elle se construit, s'enrichit et se transforme au sein d'une interaction sociale et au contact d'outils symboliques ou techniques. Ils insistent sur ce que peut faire l'élève seul et sur ce qu'il peut faire avec les autres.

\footnotetext{
${ }^{8}$ Selon Cansigno et al (2010), la compétence scripturale se développe dans des situations variées

${ }^{9}$ Selon Cansigno et al (2010), la compétence scripturale se développe continuellement
} 
Le rôle de l'enseignant est d'être dans une zone proximale ${ }^{10}$ afin de lui servir de guide ou de médiateur pour l'aider à atteindre la limite supérieure de cette zone.

Dans cette perspective, les travaux de Hall (2003), en résumant plusieurs pratiques gagnantes, mettent l'accent sur la démarche intégrée de l'enseignement de la lecture et de l'écriture ${ }^{11}$ et sur celui de l'apprentissage formel et explicite de la langue (Laroui, Morel et Leblanc, 2000). Cette démarche consiste en la mise en place de pratiques enseignantes visant le développement de la réflexion des élèves par rapport à la langue et aux stratégies ${ }^{12}$ utilisées pour comprendre et écrire des textes. Sur ce point, ces pratiques reposent sur les milieux socioculturels des élèves. Elles insistent également sur la différenciation en fonction des besoins des élèves. Cette approche favorise l'engagement de l'élève dans un contexte interactif d'apprentissage (Blain et Lafontaine, 2010) et privilégie l'enseignement explicite (Bissonnette, Richard et Gauthier, 2005) pour mener les élèves plus loin dans leurs apprentissages. Les enseignants qui envisagent le développement des compétences en écriture en classe sont censés enseigner explicitement et par application concrète des stratégies de reconnaissance de mots, le vocabulaire, l'orthographe, les stratégies de compréhension de textes, ainsi que les processus d'écriture. Cette démarche d'enseignement nous semble appropriée en ce qui a trait à l'enseignement et au développement des compétences en écriture, dans la mesure où il ne suffit pas de faire écrire les élèves pour qu'ils deviennent des scripteurs efficaces et avisés. Dans ce contexte, les plus compétents découvrent et arrivent à utiliser des stratégies, et ainsi à produire des écrits pour eux-mêmes, tandis que les autres élèves éprouvent des difficultés à ce stade de l'apprentissage de l'écriture. Par conséquent, l'écart entre les plus forts et les plus faibles s'agrandira davantage, comme c'est malheureusement souvent le cas (Giasson, 1990; Minskoff et Allsopp, 2006). Les travaux de Weinstein et Hume (2001) ont précisé que « l'enseignement direct » ou explicite peut aider les élèves à recourir à des stratégies d'apprentissage. Étant donné que le processus cognitif en lecture et en écriture n'est pas toujours accessible par tous les élèves, l'enseignant peut les aider à découvrir ces stratégies tout en les enseignant explicitement en classe. Cela aura pour effet de rendre transparents les processus impliqués et s'assurer que l'élève les maîtrise adéquatement (Giasson, 1992). D'ailleurs, dans un souci d'équité, l'enseignant est censé mettre à la disposition de tous les élèves les mêmes chances d'apprentissage et de réussite en écriture. Toutefois, l'enseignement explicite exige de l'enseignant une planification rigoureuse. Pour ceci, nous présentons les étapes de cette planification telle qu'elle a été exposée par Rosenshine (1986) :

${ }^{10}$ Ce concept est central dans les travaux de Vygotsky. Il exprime la différence entre ce que l'enfant apprendra s'il est seul, et ce qu'il peut en potentiel, apprendre si on lui fournit une aide.

${ }^{11}$ Nous considérons, dans cet article, que l'acte d'écrire est indissociable de l'acte de lire.

${ }^{12}$ Pressley et Harris (2006) définissent une stratégie comme une opération cognitive ou métacognitive complexe qui permet d'atteindre un but déterminé à l'aide d'une suite d'actions réalisées de manière consciente ou non 
Tableau 1

Les trois phases de l'enseignement explicite

\begin{tabular}{|c|c|}
\hline Phases de l'enseignement explicite & $\begin{array}{l}\text { But de chacune des phases } \\
\text { de l'enseignement explicite }\end{array}$ \\
\hline Première phase: modelage & $\begin{array}{l}\text { permet à l'élève de comprendre l'objectif } \\
\text { d'apprentissage }\end{array}$ \\
\hline Deuxième phase : pratique guidée ou dirigée & $\begin{array}{c}\text { rend possibles l'ajustement et la consolidation } \\
\text { de la compréhension pendant la réalisation } \\
\text { de l'activité }\end{array}$ \\
\hline $\begin{array}{c}\text { Troisième phase: pratique autonome ou } \\
\text { indépendante }\end{array}$ & $\begin{array}{c}\text { offre à l'élève de nombreuses occasions de } \\
\text { mettre en pratique de façon indépendante } \\
\text { ses nouvelles connaissances afin de les } \\
\text { maîtriser et de les automatiser }\end{array}$ \\
\hline
\end{tabular}

Comme le montre ce tableau, l'intervention en classe de langue, selon l'enseignement explicite de l'écriture, commence la première phase par le modelage. Dans cette phase, il est nécessaire d'expliciter verbalement ce qui se passe dans la tête d'un scripteur accompli durant le processus car les processus cognitifs ne peuvent être observés directement c'est pour cette raison qu'il importe de les décrire. Par exemple, pour faire apprendre le processus d'écriture d'un récit aux élèves, l'enseignant peut dire : « je ne sais pas par quoi je vais commencer pour écrire ce récit? Je pense qu'il faut commencer par ......, mais je ne suis pas certain. Pouvez-vous me dire par quoi vous aller commencer pour écrire ce récit? L'enseignant poursuit sur la même démarche et mentionne au fur et à mesure les éléments qui viennent expliciter la première phase du processus de l'écriture d'un récit qu'est la planification. Ceci permettra de donner aux élèves l'aide nécessaire lors des travaux pratiques. Ces travaux pratiques ont pour objectifs d'entrainer les élèves à mettre en place cette stratégie dans diverses tâches et situations. Cette pratique orientée par l'enseignant devrait leur permettre d'appliquer seuls la démarche dans des situations en pratique autonome. Pendant la deuxième phase, l'enseignant conduit les élèves à maîtriser la stratégie en donnant des indices, des rappels et en diminuant graduellement l'aide apportée. L'enseignant fournira aux élèves des commentaires sur leur façon d'utiliser la stratégie. Les élèves ont besoin de savoir pourquoi leur réponse est correcte ou non. Le travail en petit groupe permet aux élèves de voir comment les autres expliquent la stratégie et les amène à confronter leur propre conception de la stratégie. Lors de la troisième phase, l'élève assume presque toute la responsabilité du choix et de l'application de la stratégie enseignée. L'enseignant discute avec les élèves, surtout avec ceux qui ont de la difficulté, pour prévenir la cristallisation d'une application inefficace.

\section{Conclusion}

Il ressort de ce que nous avons développé ci-dessus qu'il est nécessaire de revoir nos pratiques enseignantes au regard de l'apprentissage de l'écriture et au développement des compétences scripturales. Dans un contexte où les difficultés d'apprentissage en écriture persistent en milieu scolaire, les enseignants sont de plus en plus encouragés à adopter des pratiques selon une perspective qui valorise non seulement le développement des compétences linguistiques de la langue, mais aussi les processus cognitif, métacognitif et affectif nécessaires pour produire le 
discours. Nous sommes également convaincu qu'une façon d'aider les élèves à développer leurs compétences scripturales et surtout de leur montrer l'importance de le faire, c'est d'être un modèle langagier à tout instant dans la classe, et ce, autant à l'oral qu'à l'écrit. En fait, l'unique prise en compte de la qualité des écrits lors des évaluations peut difficilement aider les élèves à développer une telle compétence dans les autres disciplines.

Nous tenons à souligner que même si cet article n'apporte pas de réponses définitives aux nombreuses questions que soulèvent l'enseignement et l'apprentissage de l'écriture, il représente, cependant, une contribution importante à la description et à la compréhension de processus mis en place par le scripteur dans une situation d'écriture. De plus, ce texte peut lever le voile sur des pratiques d'enseignement actuelles au regard de l'apprentissage de l'écriture. En ce sens, il peut participer à la discussion en cours sur les pratiques à adopter dans nos écoles afin d'améliorer l'enseignement-apprentissage de l'écriture en arabe et en langues étrangères au Maroc.

\section{Références}

[1] Adad, D. et Richard-Principalli, P. Lire pour écrire, écrire pour grandir : mener des projets d'écriture avec des enfants de 3 à 11 ans. Lyon : Chronique sociale, 2007.

[2] Altet, M. Les pédagogies de l'apprentissage. Paris: Presses universitaires de France, 1997.

[3] Association marocaine des enseignants de français. Didactique de l'écriture : contraintes et dimension créative. Agadir : Université d'été à Agadir, 2010.

[4] Arraichi, R. Environnement sociolinguistique et enseignement/apprentissage du français au Maroc. Thèse de doctorat national. Rabat : Faculté des lettres, 2002.

[5] Baratte, M.-F. (2007). La construction du rapport è l'écrit. L'écriture avant l'écriture. Paris : L'Harmattan.

[6] Blain, S. et Lafontaine, L. Mettre les pairs à contribution lors du processus d'écriture : une analyse de l'impact du groupe de révision rédactionnelle des élèves québécois et néo-brunswickois. Revue des sciences de l'éducation, 2010, 36(2), p. 469-491.

[7] Blaser, Chr. Fonction épistémique de l'écrit: pratiques et conceptions d'enseignants de sciences et d'histoire du secondaire. Université Laval, Québec. Manuscrit non publié; en ligne, 2007.

[8] Bissonnette, S., Richard, M. et Gauthier, C. Interventions pédagogiques efficaces et réussite scolaire des élèves provenant de milieux défavorisés. Revue française de pédagogie, 2003, (150), p. 87-141.

[9] Cansigno, Y., Dezutter, O. et Silva, H. Un cadre théorique pour penser le développement des compétences scripturales en langue seconde ou étrangère. Dans: Y. Cansigno. O. Dezutter. H. Silva. F. Bleys (p. 13-33). Défis d'écriture. Développer la compétence scripturale en français langue seconde ou étrangère. Universidad AutÓnoma Metropolitana, Unidad Azcapotzalco, México, 2010. 
[10] Chartrand, S.-G. Analyse comparative d'activités et de tâches prescrites pour l'écriture de textes argumentatifs au secondaire. Dans : J. Dolz, B. Schneuwly et T. Thevenaz (éd.), Les tâches et leurs entours dans la classe de français. Actes du 8e Colloque de la DFLM. Neuchâtel, Suisse, 2000a.

[11] Chartrand, S.-G. Le programme de français de 1995. Un outil pour développer la compétence d'écriture au secondaire. Québec français ( $\mathrm{n}^{\circ}$ hors-série: la grammaire au cœur du texte), 2000b, p.24-27.

[12] Commission spéciale éducation-formation. Mise en œuvre de la réforme du système d'éducation et de formation. Rabat: Gouvernement du Maroc, 1999.

[13] Conseil supérieur de l'enseignement. État et perspectives du système d'éducation et de formation: Réussir l'école pour tous. Rapport annuel, Tome 1. Rabat: Gouvernement du Maroc, 2008.

[14] Dabène, M. L'adulte et l'écriture : contribution à une didactique de l'écrit en langue maternelle. Bruxelles: De Boeck Université, 1987.

[15] Giasson, J. La compréhension en lecture. Boucherville : Gaëtan Morin éditeur, 1990.

[16] Giasson, J. Stratégies d'intervention en lecture: quatre modèles récents. Dans C. Préfontaine et M. Lebrun (Eds.), La lecture et l'écriture: enseignement et 25 apprentissage!: actes du Colloque Stratégies d'enseignement et d'apprentissage en lecture/écriture. Montréal : Éditions Logiques, 1992, 219-239.

[17] Giasson, J. La lecture. Apprentissage et difficultés. Montréal : Gaëtan Morin éditeur, 2011.

[18] Hall, K. Effective literacy teaching in the early years of school. Dans N. Hall, J. Larson et J. Marsh (dir.), Handbook of Early Chilhood literacy (315-326). London : Sage, 2003.

[19] Kesbi, A. Les problèmes de transmission et de diffusion de la langue arabe un obstacle au processus d'arabisation. San Francisco : Academia.edu, 2011.

[20] Laroui,R., Morel, M. et Leblanc, S. Des pratiques pédagogiques de l'enseignement du lire/écrire, déclarées par des enseignantes du primaire, Phronesis, 2000, (3) 1-2, 111-120.

[21] Lentin, L. Apprendre à penser parler lire écrire. Paris : ESF, 1998.

[22] Ministère de l'Éducation nationale. Programme d'urgence. Rabat: Gouvernement du Maroc, 2008.

[23] Ministère de l'Éducation, du Loisir et du Sport. Mieux soutenir le développement de la compétence à écrire. Rapport du comité d'experts sur l'apprentissage de l'écriture, Québec: Gouvernement du Québec, 2008.

[24] Minskoff, Esther et David Allsopp. Stratégies d'apprentissage et réussite au secondaire: un passeport pour les élèves en difficulté. Montréal: Chenelière Éducation, 2006 p. 307, 2006. 
[25] Olson, D. R. L'univers de l'écrit : comment la culture écrite donne forme à la pensée (traduit par Yves Bonin). Paris: Retz, 1998.

[26] Pressley, M. et Harris, K.R. Cogntive stratégies instruction : from basic research to classroom instruction. Dans P.A. Alexander et P.H. Winne (eds.), Handbood of educational psychology. Mahwah, NJ: Erlbaum, 2006, p. 265- 286.

[27] Piaget. J. Psychologie et pédagogie. Paris: Denoël-Gonthier, 1969.

[28] Prenoveau, J. Cultiver le goût de lire et d'écrire : enseigner la lecture et l'écriture par une approche équilibrée. Montréal : Chenelière Éducation, 2007.

[29] Rey, B. La notion de compétence permet-elle de répondre à l'obligation de résultats dans l'enseignement. Dans : C. Lessard et Ph. Meirieu (dir.) L'obligation des résultats en éducation Bruxelles: De Boeck Université, 2004, p. 233-242

[30] Reuter, Y. Enseigner et apprendre écrire. Paris : ESF, 1996.

[31] Reuter, Y. (2000). Enseigner et apprendre à écrire. Construire une didactique de l'écriture. Paris : ESF éditeur, 2000

[32] Reuter, Y. Penser la perspective didactique : la question de l'articulation disciplinaire, pédagogique et scolaire. Dans : B. Daunay, Y. Reuter et B. Scheneuwly (dir.). Les concepts et les méthodes en didactique du français. Namur : Presses universitaires de Namur, 2011, p. 35-54.

[33] Riegel, M., Pellat, J.-C. et Rioul, R. Grammaire méthodique du français. Paris: PUF, 2004.

[34] Routman, R. Enseigner l'écriture : revenir à l'essentiel. Montréal : Chenelière Éducation, 2009.

[35] Rosenshine. Barak V. Synthesis of Research on Explicit Teaching. Dans : Educational Leadership, 1986, vol. 43, no 7, p. 60-69.

[36] Strickland, D.-S., Ganske, K. \& Monroe, J.-K. Les difficultés en lecture et en écriture. Montréal : Chenelière Éducation, 2009.

[37] Vygotsky, L-S. Le problème de l'enseignement et du développement mental à l'âge scolaire, dans : B. Schneuwly et J.P. Bronckart, dir., Vygotsky aujour'hui, Paris, Delachaux et Niestlé, 1985, p. 113, 114.

[38] Vygotsky, L-S. Mind in society: The development of higher mental processes. Cambridge. Havard University Press, 1978.

[39] Vygotsky, L-S. Pensée et langage. (F. Sève, Trans.). Paris: La Dispute, 1997.

[40] Weinstein, Claire E. et Laura M. Hume. Stratégies pour un apprentissage durable. Paris: De Boeck Université, 2001, p. 157. 